\title{
Fluorescence visualization of a convective instability which modulates the spreading of volatile surface films
}

\author{
Anne D. Dussaud and Sandra M. Troian \\ Department of Chemical Engineering, Princeton University, Princeton, New Jersey 08544 \\ Scott R. Harris \\ Department of Mechanical Engineering, Princeton University, Princeton, New Jersey 08544
}

(Received 17 October 1997; accepted 16 March 1998)

\begin{abstract}
The spontaneous spreading of a thin liquid film along the surface of a deep liquid layer of higher surface tension is a ubiquitous process which provides rapid and efficient surface transport of organic or biological material. For a source of constant concentration, the leading edge of a nonvolatile, immiscible film driven to spread by gradients in surface tension is known to advance as $t^{3 / 4}$ in time. Recent experiments using laser shadowgraphy to detect the advancing front of spreading films indicate, however, that immiscible but volatile sources of constant concentration spread with a reduced exponent according to $t^{1 / 2}$. Using a novel technique whereby fluorescent lines are inscribed in water, we have detected the evolution of a thermal instability beneath the leading edge of volatile films which strongly resembles a Rayleigh-Bénard roll. We propose that the increased dissipation from this rotational flow structure is likely responsible for the reduction in spreading exponent. This observation suggests a conceptual framework for coupling the effects of evaporation to the dynamics of spreading. (C) 1998 American Institute of Physics. [S1070-6631(98)01607-9]
\end{abstract}

\section{SPONTANEOUS SPREADING ON A DEEP LIQUID LAYER}

When a liquid substrate is contacted by a film of lower surface tension, surface traction is produced in proportion to the difference in surface tension between the liquid support and the spreading film. This traction creates rapid and spontaneous flow toward regions of higher surface tension. The speed of the spreading film is known to increase with the magnitude of the spreading coefficient defined by $S=\gamma_{1}-\gamma_{2}-\gamma_{12}$, where $\gamma_{1}$ denotes the surface tension of the clean liquid support, $\gamma_{2}$ the surface tension of the spreading film, and $\gamma_{12}$ the interfacial tension between the spreading film and supporting liquid. ${ }^{1}$ This spreading coefficient is related to the local gradient in surface tension, $\partial \gamma / \partial x$, through the relation $S=\int_{0}^{L(t)} \partial \gamma / \partial x d x$, where the coordinate $x$ denotes the horizontal or radial direction of spreading and $L(t)$ denotes the length of the surface active film at time $t$ after contact with the liquid support. In the examples discussed below, the gradients in surface tension are caused by variations in the surface concentration, $\Gamma$, of the spreading film where $\partial \gamma / \partial x=(\partial \gamma / \partial \Gamma)(\partial \Gamma / \partial x)$. The spontaneous advance of a surface film driven by this type of shear stress is called Marangoni driven spreading, ${ }^{2}$ which occurs for positive values of the spreading coefficient. A small scale example of this spreading process occurs during the casting of Langmuir-Blodgett films. In the fabrication of these films, organic or biological molecules are dissolved in a solvent like hexane or carbon tetrachloride which spreads rapidly and spontaneously over the surface of water. The organic solvent, which has a lower surface tension than pure water, acts as the carrier liquid to transport molecules along the water surface under the action of Marangoni stresses. ${ }^{3}$ The solvent film eventually evaporates leaving behind a molecularly thin organic film which can then be compressed, lifted off the water surface and deposited onto a substrate for further processing. A large scale example of Marangoni driven spreading can be observed during the late stages of an advancing oil spill. After the contaminant oil spill has thinned sufficiently so that hydrostatic forces and inertia no longer contribute to its advance, ${ }^{4,5}$ the film will continue to spread due to gradients in surface tension between the oil mass and the ocean surface. This spreading process is responsible for the large scale contaminant "sheen" observed to extend far beyond the original periphery of the spilled oil mass.

\section{A. Leading edge of nonvolatile surface films}

Studies of the spreading of nonvolatile, immiscible films along the surface of a deep liquid layer have shown that the Marangoni stress at the air-liquid interface establishes a boundary layer in the supporting liquid whose viscous resistance controls the advance of the spreading film. A simple force balance first proposed by $\mathrm{Fay}^{4}$ determines the temporal advance of the film. Fay reasoned that for a source of constant concentration, the force per unit length driving the spreading process is the spreading coefficient, $S$. This driving force is retarded by the drag force per unit length created by the viscous stress from the boundary layer integrated over the film length $L(t)$. For a Newtonian liquid layer, the boundary layer approximation yields a surface stress proportional to $\int_{0}^{L} \mu(\partial u / \partial y)_{y=0} d x$, where $u$ represents the horizontal velocity within the supporting liquid and $y$ the depth beneath the air-liquid interface situated at $y=0$. The viscous stress at the surface can be estimated by $\mu U L / \delta$, where $U$ denotes the surface velocity of the spreading film (of order 
$L / t)$ and $\delta(t)$ denotes the viscous penetration depth which for unsteady parallel flow scales as $(\mu t / \rho)^{1 / 2}$. The quantities $\mu$ and $\rho$ represent the liquid support viscosity and density, respectively. Combining these relations produces an expression which locates the leading edge of the spreading film in rectilinear or radial geometry, namely

$$
L(t)=k \frac{S^{1 / 2}}{(\mu \rho)^{1 / 4}} t^{3 / 4} .
$$

The validity of this relation has been confirmed by numerous theoretical and experimental studies over the years. While early experiments in rectilinear geometry ${ }^{6-10}$ suggested that the prefactor $k$ is dependent on film constitutive behavior, later experiments by Camp and Berg ${ }^{11,12}$ demonstrated that this constant depends only on spreading geometry. Recent calculations ${ }^{13}$ suggest universal values of $k=1.4150$ for rectilinear spreading (in good agreement with Camp and Berg's ${ }^{11,12}$ experimental value of $1.4 \pm 0.04$ ) and $k=1.0754$ for axisymmetric spreading. The latter prediction is slightly higher than the reported value of $k=0.88$ obtained in a recent study of silicone oil drops spreading on water. ${ }^{14,15}$ The discrepancy may be related to some uncertainty in the measurement of the spreading coefficient as well as the limited number of experiments on which this experimental value is based.

The relation derived in Eq. (1) also predicts the surface velocity at the leading edge of the film, $U_{L}=d L / d t$. Detailed experiments by Camp and Berg on single component oils have clearly shown, however, that the surface velocity is not constant throughout the length of the spreading film. While a significant region behind the leading edge displays a surface velocity equal to that of the leading edge, the velocity field near the source behaves quite differently. Experimentally, a sharp transition or "acceleration zone" is observed between the source and the leading edge. The portion of the film near the source displays a characteristic stretched velocity profile where the horizontal speed increases in proportion to the distance from the source. At the transition point, the surface velocity jumps in value to the magnitude of the surface velocity at the leading edge, $U_{L}$. Depending on the type of single component oil used, the acceleration zone appears at different points between the source $(x=0)$ and leading edge $(x=L) .{ }^{11,12}$ For nonanoic acid, for example, the acceleration zone has been measured at $x=0.22 L(t)$ while $1000 \mathrm{cS}$ silicone oil displays a transition in surface velocity at $x=0.58 L(t)$. For our nonvolatile control studies using a fluorescence apparatus to be discussed, we also used $1000 \mathrm{cS}$ silicone oil for which it can be assumed that the downstream $40 \%$ portion of the film advances at the same speed as the leading edge. It has been suggested that this transition in surface velocity may be signaling a jump in film thickness from bulk-like behavior near the source to a molecularly thin film or even a monolayer closer to the leading edge. The technique of surface seeding used by Camp and Berg ${ }^{11,12}$ clearly demonstrates that the advancing molecular film behaves as a rigid plate moving with the speed of the leading edge. This specific feature of the surface velocity profile, which depends on film constitutive behavior, has not been predicted by the theoretical models developed to describe the spreading of surface active films on a deep liquid layer. ${ }^{9,10,16-18}$ Phillips has recently indicated that there do exist eigenfunctions in the spreading problem which might display this interesting jump property. ${ }^{13}$

The theoretical models that have been proposed predict certain distinctive features in the sublayer flow. In particular, we briefly describe the predicted behavior near the leading edge and near the reservoir source..$^{16,10,17,18,13}$ The unsteady laminar boundary equation describing the spreading of a surface active film on a deep layer support can be reduced to time independent form by introducing similarity variables consistent with Eq. (1) for the relevant coordinates and velocities. The calculations are not completely straightforward since the boundary layer equation is of singular parabolic form. ${ }^{18,19,13}$ In the neighborhood of the leading edge of the spreading film, the dimensionless stream function reduces to the streamfunction of a Blasius boundary layer beneath a rigid plate. Experiments indicate, however, that this behavior extends over a longer region downstream and is not just located at the tip of the spreading film. Taken together, theoretical and experimental findings suggest that a significant downstream portion of the spreading film behaves as a molecularly thin film advancing as a rigid plate whose speed equals $d L / d t$. The flow profile produced beneath the spreading film can therefore be viewed as an unsteady Blasius-like boundary layer corresponding to the flow beneath a flat plate which is stretching and travelling at a speed $d L / d t$. The surface (or "plate") speed decreases in time since the driving force for spreading, which scales as $S / L(t)$, is continuously diminished as the surface active film contaminates an ever larger area. It has also been shown that further upstream and closer to the source, the unsteady boundary layer equation experiences a reversal in the direction of the convection of vorticity. This behavior implies the formation of two boundary layers, one close to the leading edge and another close to the source. For the nonvolatile systems discussed below, we have focused strictly on the downstream portion of the spreading film known to travel at the same speed as the leading edge. In this paper, we present evidence for the analogy with an unsteady Blasius-like boundary layer beneath nonvolatile spreading films. We then describe the unusual flow field produced beneath spreading volatile films and link the structure observed to a thermal instability produced by evaporative surface cooling.

\section{B. Leading edge of volatile surface films}

The theoretical model, which appears in the literature, describing the spontaneous spreading of a thin liquid film on a deep liquid layer assumes nonvolatile, immiscible spreading films. In many practical applications, however, spreading films often contain volatile and/or soluble components. Further understanding of the coupling between evaporation and/or dissolution and the dynamics of spreading are required in order to extend the original formulation. Recently we initiated an experimental program designed to incorporate these mechanisms into a self-consistent model for spreading. Our first set of experiments have focused on the 


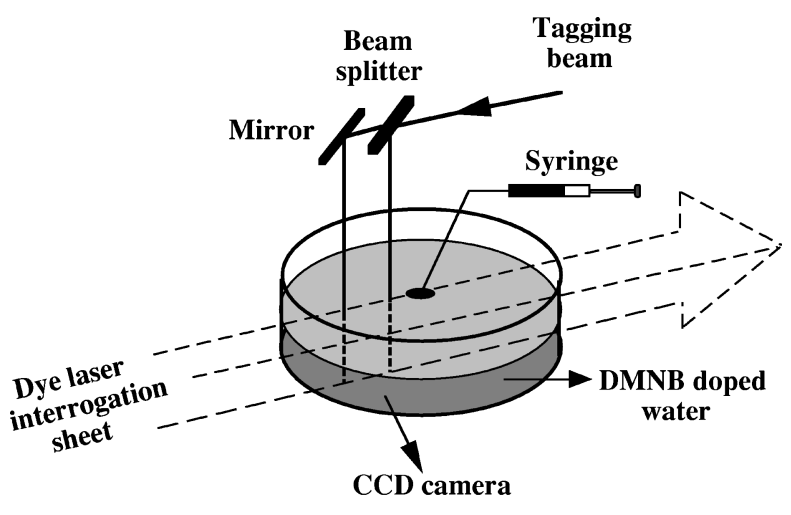

FIG. 1. Optical arrangement for flow tagging velocimetry.

dynamic behavior of volatile but immiscible hydrocarbon films spreading on a deep water layer. In lieu of surface seeding techniques to locate the position of the leading edge, we have employed laser shadowgraphy to identify several regions of the spreading film which display strong surface curvature, including the region at the advancing front. ${ }^{14,15}$ These experiments were conducted at room temperature in unsaturated conditions in order to enhance the effect of evaporation. The results show that volatile, immiscible films also advance on a deep liquid layer with a power law in time but with a reduced spreading exponent of $1 / 2$ and a weaker dependence on the spreading coefficient than described by Eq. (1). Since all the liquids tested displayed a similar reduced exponent (despite the fact that $S$ and the vapor pressure were different), we have hypothesized that the subsurface flow, which controls the rate of viscous dissipation, might be similar for each liquid studied. To confirm this possibility, another optical technique is required in order to investigate the flow behavior beneath a spreading volatile film.

\section{FLOW TAGGING VELOCIMETRY MEASUREMENTS}

Flow tagging velocimetry is a useful tool for visualizing and quantifying hydrodynamic flows. Lempert et al. ${ }^{20}$ have recently developed an optical flow tagging technique that utilizes highly water soluble, caged dye photoactivated fluorophores (PAFs). Upon exposure to photons in the range $0.350 \mu$, the caging group of the compound DMNB dextran caged carboxy fluorescein (anionic, 3000 MW, Molecular Probes, Inc.) is photolytically cleaved to recover the original fluorescein molecule which is then tracked using ordinary laser sheet fluorescence imaging. This technique, which has been used in several proof of concept studies ranging from Poiseuille flow to vortex rollup, ${ }^{20}$ is similar to dye visualization except that any desired pattern can be precisely written into the bulk water by the laser tagging geometry. The principal advantage of tracer PAFs is the simultaneous high contrast and high intensity of the images achieved with very low dye concentration. This feature is especially important in our surface studies because the dye tracer must not modify the value of the spreading coefficient since that would ultimately affect the spreading speed and therefore the thickness of the boundary layer formed. In Fig. 1 is shown a schematic of the optical apparatus used for the flow tagging measurements. The third harmonic of a Q-switched Nd:YAG laser at 0.355 $\mu$ was used to tag the water support by shining a beam from the YAG laser vertically through the water layer and "writing" one or two vertical lines in the water. The YAG laser delivered approximately $30 \mathrm{~mJ}$ per pulse. A single beam was split into two beams of $15 \mathrm{~mJ}$ when needed. The fluorescent lines were interrogated by a vertical laser sheet produced by a $5 \mathrm{~Hz}$ flashlamp pumped dye laser (LD-490 laser dye) which excites the dye molecules at $0.490 \mu$ to fluoresce at $0.510 \mu$. The dye laser delivered approximately $200 \mathrm{~mJ}$ per pulse. As shown in Fig. 1, the laser sheet was oriented in the plane defined by the tagged lines in order to capture the radial displacement of the dye lines as a function of time. Since light distortion through the curved walls of the glass tank amounted to less than one pixel across the field of interest, no ray tracing was required in reconstructing displacement and velocity profiles.

The experiments were conducted in a circular transparent glass tank measuring $8 \mathrm{~cm}$ in radius and depth. Appropriate degreasing and cleaning measures were undertaken for the glass tank and all parts in contact with the water or syringe delivery system as previously described. ${ }^{14,15}$ The tank was filled to a depth of $4 \mathrm{~cm}$ with an aqueous dextran carboxy fluorescein solution $(0.5 \mathrm{mg} / \ell)$. The addition of this small amount of caged fluorescein did not affect the surface tension of the ultrapure water (distilled and deionized 18 $\mathrm{M} \Omega$ ) which was measured to be $72.2 \pm 0.3 \mathrm{dyn} / \mathrm{cm}$ at $23{ }^{\circ} \mathrm{C}$. To avoid any forceful ejection and possible splatter of a test droplet onto the water surface (which could introduce undesirable inertial effects during the spreading process), the droplet was carefully brought into contact with the water surface by translating a syringe needle attached to a micrometer driven vertical translation stage. As soon as the droplet touched the water surface, it was rapidly wicked away and spread across the surface of the deep water layer. This thin spreading film was observed to shear the tagged lines and displace them radially. The location of the displaced lines was recorded by a high resolution black and white Dage CCD camera fitted with a Navitar TV zoom lens. The images were stored onto SVHS tapes and analyzed using standard image processing software.

\section{A. Spreading behavior of nonvolatile surface films}

Since this flow tagging device has never before been used in studies of the dynamics of spreading, the apparatus was first tested by measuring the velocity profile in the supporting water layer during the spreading of a nonvolatile, immiscible film. Silicone oil DC 200 (Fluka, $S=9.4 \pm 0.2$ $\mathrm{dyn} / \mathrm{cm}, \mu=965 \mathrm{cp}, \rho=0.965 \mathrm{~g} / \mathrm{cm}^{3}$ at $25^{\circ} \mathrm{C}$ ) was chosen as the control sample. As discussed in Sec. I A, previous studies have shown that beyond a distance $x=0.58 L(t)$ from the origin, the surface velocity is spatially uniform and equal to that of the leading edge. We measured the subsurface velocity profile beneath this "rigid" portion of the film in order to confirm the analogy with an "unsteady" Blasius boundary layer.

A microliter Hamilton glass syringe was used to deposit 


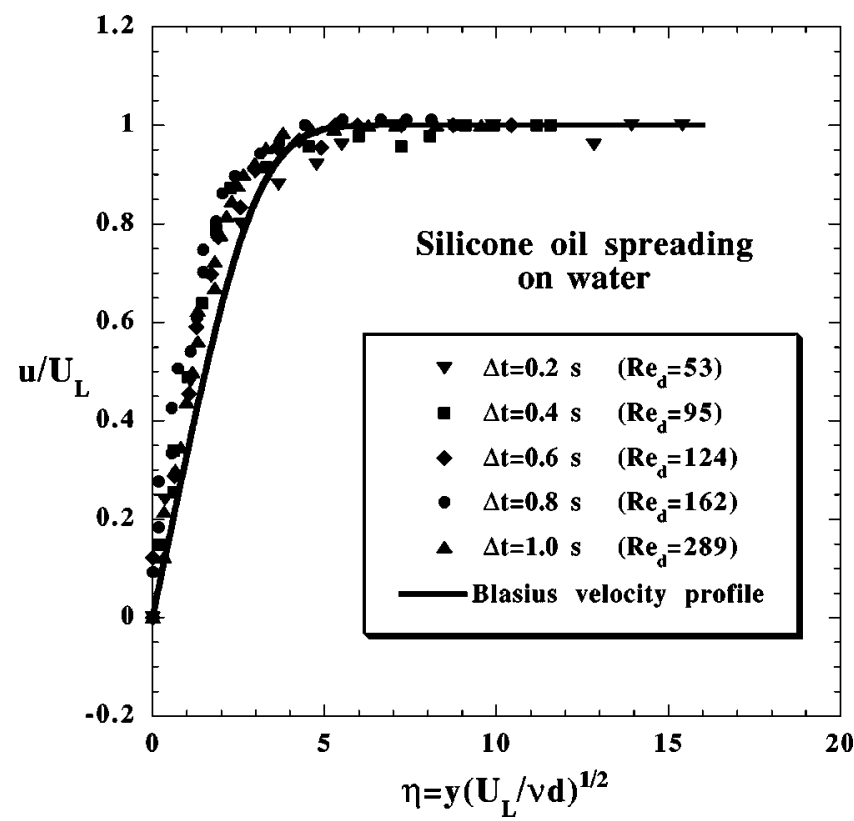

FIG. 2. Dimensionless velocity profile in water sublayer for silicone oil spreading on water. Icons represent experimental data obtained at five successive intervals after motion of tagged line commences. All measurements correspond to section of spreading film known to behave as a rigid monolayer. Solid line represents theoretical profile for Blasius boundary layer with free stream velocity equal to $U_{L} \approx d / \Delta t$.

a $3 \mu \ell$ droplet of silicone oil onto the quiescent water surface. Our previous studies ${ }^{15}$ have shown that during the first few seconds of spreading, such small volumes still behave as a source of constant concentration to produce a film that advances according to the $3 / 4$ spreading law. A single vertical fluorescent line was drawn at a distance of $4.24 \mathrm{~cm}$ away from the point of deposition. The horizontal displacement of the entire fluorescent line as a function of the depth beneath the air-liquid surface was measured at five successive time intervals after motion of the tagged line commenced, namely $\Delta t=0.2,0.4,0.6,0.8$ and $1.0 \mathrm{~s}$. (The pumped dye laser did not allow us to probe time intervals smaller than $0.2 \mathrm{~s}$.) This task was performed by digitizing the relevant image and using a mouse driven cursor to trace out the $(x, y)$ coordinates of the pixels corresponding to the displaced fluorescent line. In order to nondimensionalize the velocity field, a measurement of the average surface velocity of the film was required corresponding to each time interval $\Delta t$. An estimate of the surface velocity was obtained by measuring the displacement, $d$, of the fluorescent element at the air-liquid interface from its original undisturbed position and dividing by the appropriate time interval $\Delta t$. This estimate, $d / \Delta$, should provide a reliable measure of $U_{L}=d L / d t$ for short time intervals after motion of the dye line commences. For the longer time intervals, this estimate provides an average speed which slightly underestimates the actual value of the surface velocity [since the surface speed of the forward part of the film is continuously decreasing according to Eq. (1)].

In Fig. 2 is shown the resulting dimensionless velocity profile throughout the water sublayer, $u / U_{L}$, as a function of the dimensionless depth below the surface, $\eta=y\left(U_{L} / \nu d\right)^{1 / 2}$, for a typical spreading run of silicone oil
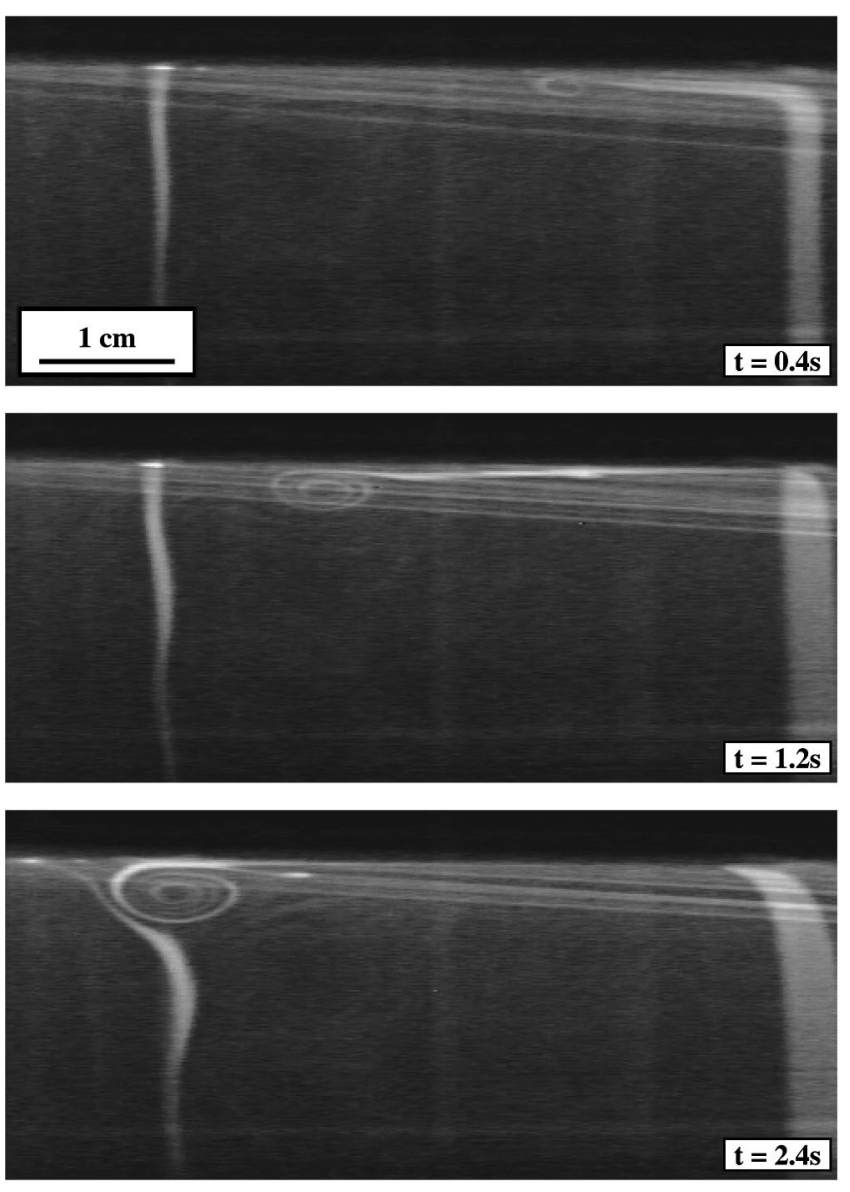

FIG. 3. Fluorescence images of sublayer flow for $p$-xylene spreading on water at three instances in time after droplet deposition. Rotational motion is clearly evident beneath leading edge of spreading volatile film.

on water. The quantity $\nu=\mu / \rho$ represents the kinematic viscosity of the water. These dimensionless variables were chosen to make comparison with the Blasius velocity profile produced for steady uniform flow over a thin flat plate, which is plotted as a solid line in Fig. 2. The effective Reynolds number for the displaced tagged line has been defined as $\operatorname{Re}_{d}=\rho U_{L} d / \mu$. All five measurements were taken within the "rigid plate" regime close to the leading edge. Even for the longest time interval accepted ( $\Delta t=1.0 \mathrm{~s})$, however, the dye line only traveled a distance $d=0.29 L(t)$, well within the downstream $40 \%$ portion of the film known to advance at the speed of the leading edge. According to Fig. 2, there is good agreement between theory and experiment. This agreement establishes that a nonvolatile, immiscible film spreading spontaneously over the surface of a quiescent deep layer gives rise to an unsteady Blasius-like velocity profile near the downstream portion of the film. This agreement also confirms that the subsurface flow is mostly unidirectional in character. Below we describe the results of experiments using volatile films which signal the breakdown of the boundary layer approximation beneath the leading edge.

\section{B. Spreading behavior of volatile films}

Volatile films undergoing evaporation while spreading produce a very different subsurface signature. Figure 3 is a 
time evolution photograph of the displacement profile of two initially vertical dye lines during the spreading of a $3 \mu \ell p$-xylene droplet on water. The hydrocarbon $p$-xylene $(99 \%+$, Aldrich) has a vapor pressure of $8.7 \mathrm{~mm} \mathrm{Hg}$, a spreading coefficient on water of $S=7.4 \pm 0.2 \mathrm{dyn} / \mathrm{cm}$, and density $\rho=0.857 \mathrm{~g} / \mathrm{cm}^{3}$ at $25^{\circ} \mathrm{C}$. The time stamps on the three frames shown, $t=0.4,1.2$ and $2.4 \mathrm{~s}$, correspond to the time elapsed after droplet deposition (not to be confused with $\Delta t$ defined earlier). Experiments conducted with toluene (99.8\%, Aldrich), which has a higher vapor pressure $(30 \mathrm{~mm}$ $\mathrm{Hg})$, a slightly larger spreading coefficient $(S=8.2 \pm 0.2$ dyn $/ \mathrm{cm}$ ), and density $\rho=0.862 \mathrm{~g} / \mathrm{cm}^{3}$ at $25^{\circ} \mathrm{C}$, displayed the same general behavior as shown in Figs. 3 and 4. The horizontal boundary near the top of each frame in Fig. 3, which separates the black from the gray region, represents the air-liquid interface along which the volatile film spreads from right to left. The syringe needle used to deposit the liquid droplet is located to the right and out of view. The slanted striations below the air-liquid interface are artifacts introduced by reflections of the laser sheet from the cylindrical lip of the glass tank. The fluorescent line to the right is somewhat wider in shape because of the lens arrangement that was used to write the lines. The optical assembly could be improved to produce equal width lines. The wider line, which is displaced first because of its proximity to the point of deposition, is strongly bent into an elbow shape and then pulled in the horizontal direction. After approximately $0.4 \mathrm{~s}$, the tip of the tagged line begins to wind around an imaginary axis oriented perpendicular to the page. The number of windings appears to increase with time indicating a region of fluid circulation which propagates radially. From above, this flow structure would resemble a propagating torus whose vertical cross section increases in time. The second vertical line is displaced to the left slightly in advance of actual contact with the propagating roll. The leading edge of the spreading film must therefore extend a small distance beyond the roll structure. Inspection of the third frame, which presents a well developed roll, reveals that the lower portion of the second line is displaced to the right as expected for rotational flow.

Since the dye lines are observed to move vertically as well as horizontally, it is no longer possible to infer directly from their displacement the velocity profile in the water layer. Fluid elements located at an original horizontal position beneath the air-liquid interface suffer translation and rotation during the spreading process. The only available information that can be obtained from frames such as those shown in Fig. 3 is the spatial location of the stretched and rotated dye line. The filled circles in Fig. 4 represent the digitized contours of the leftmost edge of the dye line closest to the point of deposition for $\Delta t=0.2,0.4$ and $0.6 \mathrm{~s}$ after motion of the line has commenced. For the series shown in Figs. 3 and 4, the dye line was located at a distance $1.66 \mathrm{~cm}$ from the point of deposition. At the final time shown $(\Delta t=0.6 \mathrm{~s})$, the displacement of the tagged line from its undisturbed position at the air-liquid interface was measured to be $d=2.70 \mathrm{~cm}$ while the length of the spreading film from the origin was measured to be $L=4.27 \mathrm{~cm}$. The final measurement in this series was therefore made at a location from the origin corresponding to $x=(4.27$
$-2.70) / 4.27 L(t) \approx 37 \% L(t)$. While for nonvolatile films this position might lie outside the region corresponding to the rigid plate approximation, for volatile films it is unknown whether there exists an equivalent acceleration zone and where it might be located. As a point of comparison, however, no region of large scale circulation was ever observed in the silicone oil studies even for times beyond $\Delta t=1.0 \mathrm{~s}$ when $d / L$ far exceeded $40 \%$ and the rigid plate approximation was no longer valid. Aside from the fact that toluene spreads more rapidly than $p$-xylene on water, the displacement profiles for toluene bear the same distorted shape as the profiles shown in Fig. 4.

The surface velocity near the downstream portion of the spreading volatile film was estimated as described earlier by measuring the quantity $d / \Delta t$. These values agreed very well with previous measurements of the speed of the leading edge, $d L / d t$, obtained by laser shadowgraphy. ${ }^{14,15}$ Besides simply digitizing the displaced dye lines, it is also useful to make comparison with the displacement that would be observed beneath a nonvolatile film traveling at the same speed as the leading edge of the $p$-xylene film. From the estimated surface velocity of the volatile film, we calculated the Lagrangian position, $[x, y]=[u \Delta t, y]$, that would instead have resulted had the sublayer flow been purely unidirectional and Blasius-like, as for the silicone oil spreading on water. The solid lines shown in Fig. 4 represent the theoretical displacement profiles for a nonvolatile film spreading with the same surface speed as the volatile $p$-xylene film. It is evident from these curves that the actual fluid displacement beneath a volatile film is inconsistent with the shear field produced by an unsteady Blasius boundary layer. The experimental data trace out a very flat trajectory ahead of the original location of the dye line whose foremost region undergoes substantial rotation. The dye line undergoes strong deformation as it is pulled upward into a tight $90^{\circ}$ bend and then stretched in the horizontal direction. This flow structure obviates the possibility of using the simple boundary layer approximation, which led to the $3 / 4$ spreading exponent for non-volatile films, to predict the $1 / 2$ exponent observed with volatile systems.

A key to the rotational structure observed in the fluorescence experiments lies with the temperature information contained in the sheared water layer. The water layer beneath a $p$-xylene or toluene film spreading in unsaturated conditions was observed to undergo a cooling effect during the spreading process. Density variations occurring in the bulk water during the rapid surface spreading were visualized by a laser shadowgraphy apparatus in which an incident He-Ne laser beam was directed to shine horizontally through one side of a water filled rectangular glass tank. Further details of the apparatus can be found elsewhere. ${ }^{14,15}$ Sideview shadowgraphs taken with this arrangement highlight regions in the bulk water which undergo density variations due to thermal or compositional effects during the surface spreading process.

In Fig. 5 is shown three shadowgraphs obtained at times $t=0.9,1.6$, and $2.7 \mathrm{~s}$ after deposition of a $3 \mu \ell$ droplet of $p$-xylene on water. The horizontal black line running across the field of view near the top of each frame represents the top 

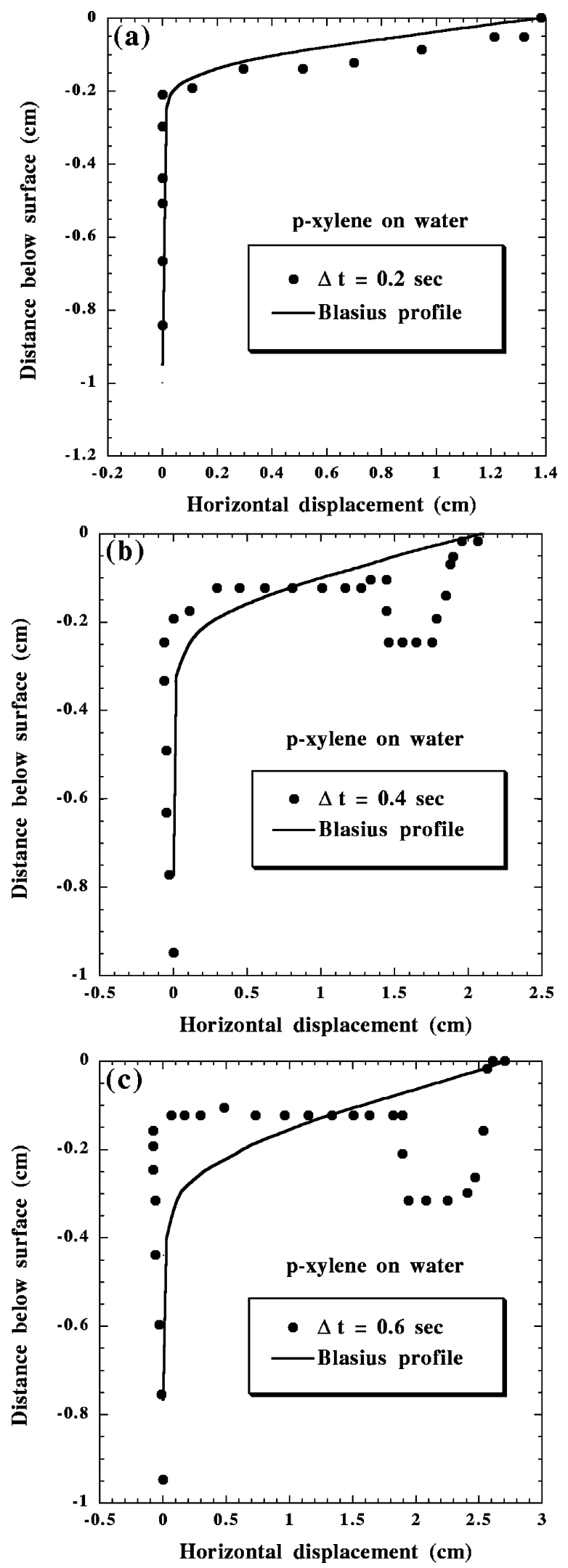

FIG. 4. Digitized displacement profiles corresponding to a fluorescently tagged line sheared during the surface spreading of $p$-xylene spreading on water. The foremost part of the profiles beneath the leading edge of the spreading film reflects the contour of the region in the water layer undergoing rotational flow. The solid line, shown for comparison, corresponds to the Lagrangian displacement that would be produced by a simple Blasius-like flow beneath a nonvolatile surface film traveling at the same surface velocity as the volatile $p$-xylene film.
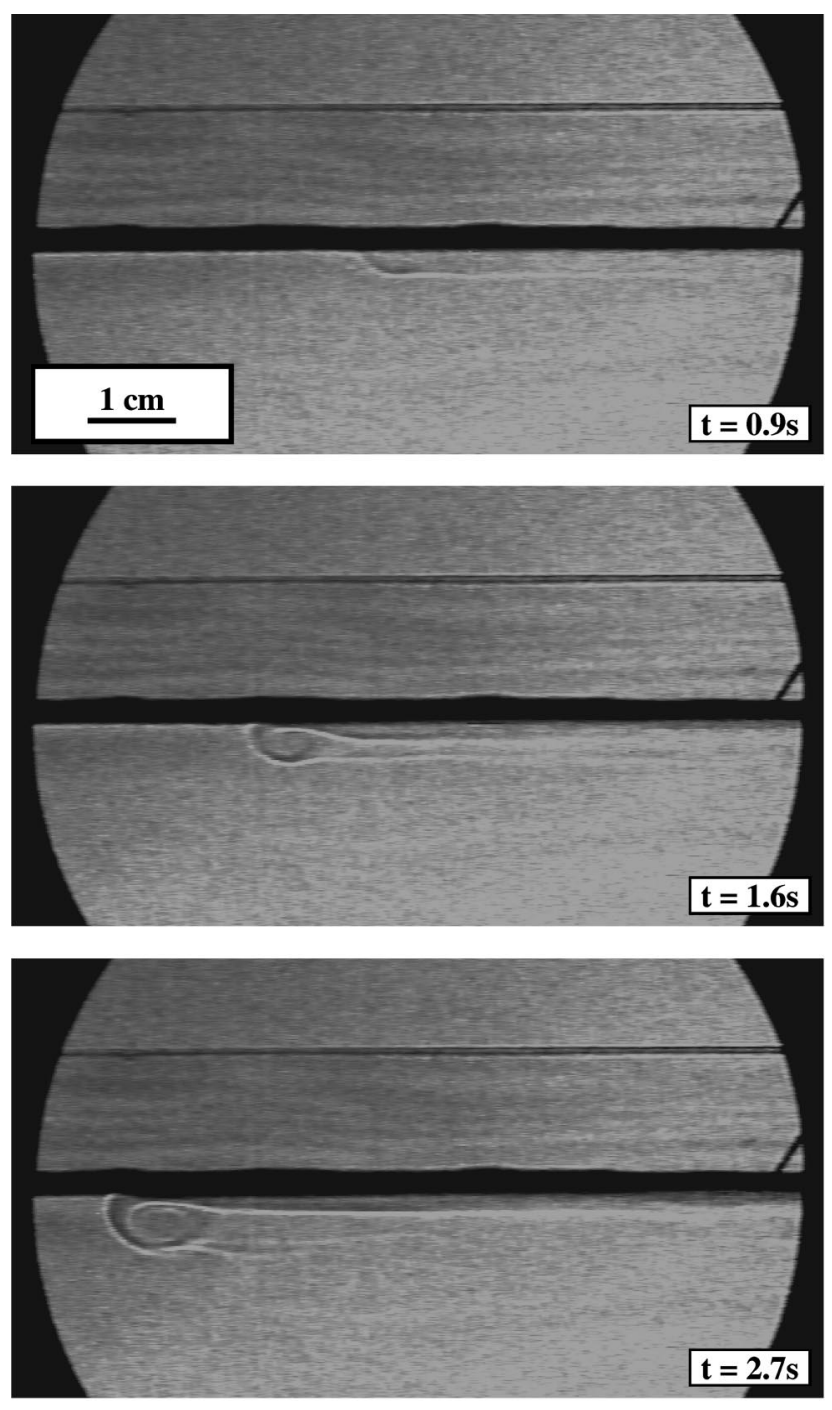

FIG. 5. Laser shadowgraphy images of sublayer flow for $p$-xylene spreading on water at three instants in time after droplet deposition. Layered structure below air-water interface highlights regions which develop density variations due to thermal effects. Elliptical shape to the left of the layered region demarcates region of fluid circulation.

lip of the glass tank. The thicker black horizontal band in the center of each photograph represents the region of light deflection caused by the water meniscus on the inside surface of the glass tank. The bottom of this band locates the actual air-water interface. The bright lines which form delineate a shear water layer which undergoes visible density changes during the spreading process. The foremost region of this layer develops an elliptical roll which can be identified as the same region of fluid circulation beneath the spreading film previously shown in Figs. 3 and 4. Measurements of the aspect ratio of the elliptical cross section from the shadowgraphs compare well with those from the shadowgraphy experiments indicating that both techniques are probing the same convective structure. As can be seen from the length scale on the shadowgraphs in Fig. 5, the visible layer which develops beneath the spreading film measures several millimeters in thickness. Compositional variations can therefore be ruled out as the main source of light deflection since the surface film is immiscible with water and displays colored 


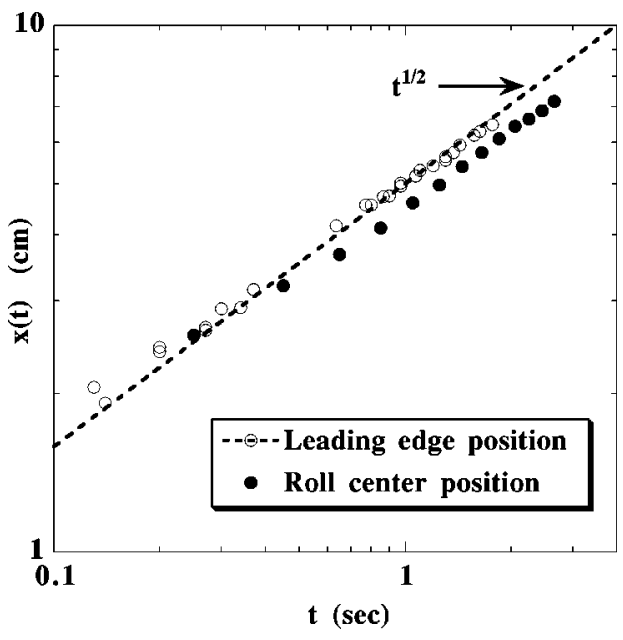

FIG. 6. Comparison of the temporal advance of the leading edge and the center of the elliptical roll for $p$-xylene spreading on water. Data for the leading edge were obtained from laser shadowgraphy (open circles) while data for the center of the roll were obtained from fluorescence flow tagging (filled circles). The dotted line represents a spreading exponent of $1 / 2$

fringes while spreading, implying that the film thickness is in the micron range. The highly visible sheared layer in Fig. 5 is therefore linked to the evolution of a cooled horizontal layer which lies just below the air-liquid interface. No such large scale cooled region was ever detected with this apparatus during the spreading of silicone oil on water. In Fig. 6 we compare data obtained previously with laser shadowgraphy ${ }^{14,15}$ to locate the leading edge of $p$-xylene spreading on water with data obtained from the fluorescence flow tagging to map the position of the center of the elliptical roll which develops beneath the same spreading system. The open circles represent the location of the leading edge obtained from two different spreading events, while the filled circles represent the location of the center of the roll for the sequence shown in Fig. 3. The data indicate that the convective roll develops beneath the advancing front of the spreading film and propagates radially at the same speed as the leading edge of the film. Although the optical field of view of the two different apparati only captures the spreading process over a short time period, both the center of the ellipse and the leading edge of the spreading film advance in time as $t^{1 / 2}$, as indicated by the dotted line.

\section{CHARACTERISTICS AND PROBABLE ORIGIN OF THE PROPAGATING CONVECTIVE ROLL}

The flow tagging profiles shown in Figs. 3 and 4 and the thermal signature shown in Fig. 5 reveal the development of a convective roll beneath the leading edge of a volatile film spreading spontaneously and rapidly across the surface of a deep water layer. We link this flow structure to the development of a thermal instability during the spreading process. It is well known that a thin quiescent liquid layer subject to a vertical temperature gradient can undergo Rayleigh-Bénard convection when the lower supporting surface is held warmer than the top surface of the liquid ${ }^{21,22}$ if the critical Rayleigh or Marangoni number is exceeded. When viewed from the top, for example, a liquid layer contained in a cir- cular cell will spontaneously produce either a series of concentric rolls coincident with the curved sidewalls or cellularlike hexagonal patterns. Our studies with volatile films spreading in an "open" axisymmetric geometry reveal only a single radially propagating, transverse roll. We have never observed a series of convective rolls nor a hexagonal convective pattern (except during a dewetting process which occurs at very late times ${ }^{23}$ ). Despite the lack of fixed restraining sidewalls as in the usual Rayleigh-Bénard problem, the leading edge of the film seems to provide a natural moving boundary which confines the convective roll. In analogy with the classic Rayleigh-Bénard instability, it appears that the observed roll develops spontaneously in response to a vertical temperature gradient created by evaporative surface cooling. The convective roll does not appear immediately upon deposition of the volatile droplet on the water surface but requires the surface film to spread some distance. This nascent period may be related to the fact that a critical vertical temperature gradient must first be established before the convective instability can be produced. This is substantiated by experiments with films more volatile than $p$-xylene for which the roll develops earlier in the spreading process.

By considering the simplest possible convectivediffusive model, ${ }^{24}$ we can understand the source and location of the instability during the spreading process. If we assume to first order that the spreading coefficient is unaffected by the evaporative mass loss and that the forward part of the spreading film can be treated as a flat plate undergoing simultaneous spreading and evaporation, then the leading edge of the spreading film will undergo the strongest cooling effect since it experiences the largest evaporative flux. The vertical temperature difference between the warmer bulk water and the cooler liquid surface is therefore expected to be largest at the leading edge. According to this simple convective-diffusive model, the concentration boundary layer established in the air close to the air-liquid interface should cause the evaporative flux to diminish away from the leading edge. The upstream portion of the spreading film will then be warmer than the downstream portion. If this is indeed the case, then the evaporating surface will create a spontaneous vertical and horizontal temperature gradient in the sheared water layer. A quantitative measure of these temperature gradients is required to confirm this picture. Unfortunately, direct thermocouple measurements in the overlying concentration boundary layer and the subsurface sheared layer have proved difficult and inconclusive. We suspect, nonetheless, that the reason that the convective roll appears beneath the leading edge is related to the fact that the leading edge of the film is first able to exceed the critical temperature drop required for onset of thermal convection.

Partly due to the difficulty in making direct temperature measurements, it cannot yet be determined whether this thermal instability is caused by buoyancy forces (Rayleigh convection), thermocapillary forces (Bénard convection), ${ }^{25}$ or both acting in conjunction. Because the thickness of the sheared layer which participates in the rotational flow is of the order of several millimeters, both effects are expected to contribute to the roll formation. The relevant critical Ray- 
leigh number $\left(\mathrm{Ra}_{c}\right)$ and Marangoni number $\left(\mathrm{Ma}_{c}\right)$ for the volatile spreading system described in this paper is, of course, not available in the literature. Rough estimates of the temperature drop that would be required to produce an instability can be made based on the values that have been derived in the literature for free-free boundaries and no shear $^{26}$ $\left(\mathrm{Ra}_{c}=658\right)$ or for a lower rigid surface, a free upper surface, and no $\left.\operatorname{shear}^{27}\right)\left(\mathrm{Ma}_{c}=80\right)$. These estimates give values of $\Delta T \approx 1.8{ }^{\circ} \mathrm{C}$ for buoyancy driven flow and $\Delta T \approx 0.03{ }^{\circ} \mathrm{C}$ for thermocapillary driven flow ${ }^{15}$ where the layer depth is the thickness of the cooled boundary shown in Fig. 5 and not the depth of the entire water support. Although the boundary conditions corresponding to these critical numbers are not the appropriate ones to use for the spreading problem, the estimates are useful for illustrating the order of magnitude in temperature drop required to initiate unstable flow.

The critical numbers cited above were originally derived for the case of a linear vertical temperature profile. The possibility of both a vertical and horizontal temperature gradient is expected to produce a nonlinear temperature profile in the supporting water layer. The calculation corresponding to a nonlinear temperature field applied to a liquid layer undergoing simple shear has only recently been presented for flow in a closed system. ${ }^{28}$ Earlier theoretical work ${ }^{29}$ has shown that the critical Rayleigh number for instability can be reduced up to a factor of 6 when a quiescent liquid layer (i.e., no imposed shear field) contained in a long horizontal rigid container is subject to a vertical and horizontal temperature gradient established by holding the bottom and left boundaries at a warmer temperature than the top and right boundaries. This result has been explained by noting that the horizontal temperature gradient will tend to enhance the rotational flow thereby lowering the critical value required for onset of convection. Theoretical studies have also shown that a gentle Couette or Poiseuille flow subject to a linear vertical temperature gradient will undergo instability in classic Rayleigh-Bénard fashion with the relevant critical values unchanged. ${ }^{30}$ Gentle shear merely serves to orient the longitudinal or transverse convective rolls when formed. Significant deviations from the classic description occur if in addition to an external flow field and a vertical temperature gradient, there exists a horizontal temperature gradient. A horizontal temperature gradient will produce thermal advection and a nonlinear temperature profile. ${ }^{31}$ As discussed by Stiller and Schöpf, ${ }^{28}$ there have been very few studies of this sort in an open geometry. Their recent studies (for no-slip upper and lower boundaries and constant heat flux) suggest that the nonlinear temperature profile can trigger a small unstable layer unrelated to the actual channel height, as indeed we have observed for volatile films. The modes they investigated, however, were strictly longitudinal.

\section{CONCLUSION AND DIRECTIONS FOR FUTURE STUDY}

By combining results from fluorescence flow tagging and laser shadowgraphy, it is evident that the flow field beneath the leading edge of a volatile spreading film bears no resemblance to the unsteady Blasius boundary layer formed beneath nonvolatile films. Rapid surface spreading in conjunction with evaporation generates a cool shear water layer beneath the spreading film. The drop in surface temperature is expected to be largest near the leading edge where a thermal instability linked to Rayleigh-Bénard convection has been observed. The instability resembles a single transverse propagating roll. The rotational flow within the propagating roll must produce excess viscous dissipation not present in the boundary layer created beneath nonvolatile spreading films. This additional source of dissipation should certainly contribute to the deceleration of a volatile spreading film. We expect that this excess dissipation is ultimately responsible for the smaller spreading exponent that has been measured in volatile spreading films. The exact value of $1 / 2$, however, may be due to the thermal instability as well as the presence of a time dependent spreading coefficient created by a nonuniform evaporation rate, as expected from a simple convective-diffusive model for spreading.

In this work we have addressed the spreading dynamics of volatile and immiscible films. Another fruitful area of investigation is the study of nonvolatile but highly miscible, spontaneously spreading films. Given the analogy between thermally driven and solutal driven instabilities, ${ }^{25}$ it is tempting to speculate that the thermal instabililty observed beneath volatile surface films could be paralleled by the formation of solutal instability beneath nonvolatile but highly miscible surface films. The presence of a vertical concentration gradient would play the same destabilizing role as a vertical temperature gradient. Each of these processes can produce convective rolls. Recent experiments ${ }^{32}$ involving the spreading of highly miscible nitroethane films in a saturated atmosphere spreading on the surface of a deep liquid layer consisting of a mixture of nitroethane and water have also found that the surface film advances with an exponent close to $1 / 2$. Schlieren studies of the subsurface flow have shown significant fluid circulation beneath the leading edge. This apparent parallelism between volatile and soluble systems may offer a tantalizing clue into the general mechanism for convective instability whose surface signature appears to be the $1 / 2$ spreading exponent.

\section{ACKNOWLEDGMENTS}

The authors would like to thank Dr. W. R. Lempert and Professor R. B. Miles for allowing us access to the flow tagging apparatus. S.M.T. gratefully acknowledges support from the NSF through its RIA and CAREER programs as well as a seed grant distributed through the Princeton Materials Institute. The Exxon Education Foundation and the Can Manufacturer's Institute also provided funding for this work.

${ }^{1}$ W. D. Harkins, The Physical Chemistry of Surface Films (Reinhold, New York, 1952).

${ }^{2}$ C. G. M. Marangoni, "Sul principio della viscosità superficiale dei liquidi stabilitito dal Sig. J. Plateau,' Nuovo Cimento 2, 239 (1872).

${ }^{3}$ A. Gericke, J. Simon-Kutscher, and H. Huhnerfuss, "Influence of the spreading solvent on the properties of monolayers at the air/water interface," Langmuir 9, 2119 (1993).

${ }^{4}$ J. A. Fay, in Oil on the Sea, edited by D. Hoult (Plenum Press, New York, 1969). 
${ }^{5}$ D. Hoult, "Oil spreading on the sea," Annu. Rev. Fluid Mech. 4, 341 (1972).

${ }^{6}$ E. Landt and M. Volmer, " "Über die Ausbreitungsgeschwindigkeit von Öl auf Wasser,' Z. Phys. Chem. (Leipzig) 122, 398 (1926).

${ }^{7}$ W. D. Garrett and W. R. Barger, "Factors affecting the use of monomolecular surface films to control oil pollution on water,' Environ. Sci. Technol. 4, 123 (1970).

${ }^{8}$ R. A. S. Lee, "A study of the surface tension controlled regime of oil spread," Master's thesis, MIT, 1971.

${ }^{9} \mathrm{C}$. Huh, M. Inoue, and S. Mason, "Unidirectional spreading of one liquid on the surface of another," Can. J. Chem. Eng. 53, 367 (1975).

${ }^{10} \mathrm{M}$. Foda and R. Cox, "The spreading of thin liquid films on a water-air interface," J. Fluid Mech. 101, 33 (1980).

${ }^{11}$ D. W. Camp, Ph.D. thesis, Dept. of Chem. Eng, University of Washington, 1985.

${ }^{12}$ D. W. Camp and J. Berg, "The spreading of oil on water in the surface tension regime," J. Fluid Mech. 184, 445 (1987).

${ }^{13}$ W. R. C. Phillips, "On the spreading radius of surface tension driven oil on deep water," Appl. Sci. Res. 57, 67 (1997).

${ }^{14}$ A. D. Dussaud and S. M. Troian, in Dynamics in Confining Systems III, edited by J. Drake, J. Klafter, and R. Kopelman (Materials Research Society, Pittsburgh, PA, 1997), Vol. 464.

${ }^{15}$ A. D. Dussaud and S. M. Troian, "Dynamics of spontaneous spreading with evaporation on a deep fluid layer," Phys. Fluids 10, 23 (1998).

${ }^{16}$ N. D. DiPietro, C. Huh, and R. Cox, "The hydrodynamics of the spreading of one liquid on the surface of another," J. Fluid Mech. 84, 529 (1978).

${ }^{17}$ Z. Dagan, "Spreading of films of adsorption on a liquid surface," Physico-Chem. Hydrodyn. 5, 43 (1984).

${ }^{18} \mathrm{O}$. E. Jensen, "The spreading of insoluble surfactant at the free surface of a deep fluid layer," J. Fluid Mech. 293, 349 (1995).

${ }^{19}$ W. R. C. Phillips, "On a class of unsteady boundary layers of finite extent," J. Fluid Mech. 319, 151 (1996).
${ }^{20} \mathrm{~W}$. Lempert et al., "Flow tagging velocimetry in incompressible flow using photo-activated nonintrusive tracking of molecular motion," Exp. Fluids 18, 249 (1995).

${ }^{21} \mathrm{H}$. Bénard, "Les tourbillons cellulaires dans une nappe liquide," Rev. Gen. Sci. Pures Appl. 11, 1261 (1900).

${ }^{22}$ S. Chandrasekhar, Hydrodynamic and Hydromagnetic Stability (Dover, New York, 1961), p. 26, Chap. 2

${ }^{23} \mathrm{~S}$. M. Troian and J. M. Drake, "Pattern generation in the dewetting of volatile films from the surface of a deep water support," unpublished, 1997.

${ }^{24}$ R. B. Bird, W. E. Stewart, and E. N. Lightfoot, Transport Phenomena (Wiley, New York, 1960).

${ }^{25}$ S. H. Davis, “Thermocapillary instabilities," Annu. Rev. Fluid Mech. 19, 403 (1987), and references therein.

${ }^{26}$ E. Koschmieder, "Benard convection," Adv. Chem. Phys. 26, 177 (1974).

${ }^{27}$ J. Pearson, "On convection cells induced by surface tension," J. Fluid Mech. 4, 489 (1958).

${ }^{28}$ O. Stiller and W. Schöpf, "Thermal instability of flows with a horizontal temperature gradient," Phys. Rev. Lett. 79, 1674 (1997).

${ }^{29}$ T. Unny and P. Niessen, "Thermal instability in fluid layers in the presence of horizontal and vertical temperature gradients," J. Appl. Mech. 36, 121 (1969).

${ }^{30} \mathrm{R}$. Kelly, "The onset and development of thermal convection in fully developed shear flows," Adv. Appl. Mech. 31, 35 (1994).

${ }^{31}$ D. A. Nield, "The onset of transient convective instability," J. Fluid Mech. 71, 441 (1975).

${ }^{32}$ M. Santiago, M. Vignes-Adler, and M. Velarde, in Fluid Physics, Lecture Notes of Summer Schools, Series B, Vol. 5, edited by M. G. Velarde and C. I. Christov (World Scientific, Singapore, 1995). 